\title{
Appendix Table 1:
}

US Level Time Series Regressions of Change in Federal Taxes Per Capita, Federal Spending Per Capita, UI Spending On Change in GDP Per Capita or Change in Unemployment Rate

Notes: Years included are 1983-2010. Columns (6)-(10) exclude the Great Recessions Years of 2008-2010. Data on federal taxes, spending are taken from National Income Accounts via FRED. Unemployment Insurance (UI) spending data are from the Consolidated Federal Funds Report. "Balance" is equal to taxes minus spending.

\begin{tabular}{|c|c|c|c|c|c|}
\hline & $\begin{array}{r}(1) \\
\text { Balance }\end{array}$ & $\begin{array}{r}(2) \\
\text { taxes }\end{array}$ & $\begin{array}{r}\text { (3) } \\
\text { spending }\end{array}$ & $\begin{array}{r}(4) \\
\text { UI_spending }\end{array}$ & $\begin{array}{r}(5) \\
\text { UI Spending }\end{array}$ \\
\hline $\begin{array}{l}\text { Change in GDP per } \\
\text { Capita }\end{array}$ & $\begin{array}{r}0.833^{* * *} \\
(0.116)\end{array}$ & $\begin{array}{r}0.551 * * * \\
(0.0640)\end{array}$ & $\begin{array}{r}-0.282 * * * \\
(0.0791)\end{array}$ & $\begin{array}{r}-0.0698 * * * \\
(0.00883)\end{array}$ & \\
\hline $\begin{array}{l}\text { Change in Unemployment } \\
\text { Rate } \\
\text { Constant }\end{array}$ & $\begin{array}{r}-1,106 * * * \\
(156.6)\end{array}$ & $\begin{array}{r}-490.9 * * * \\
(86.31)\end{array}$ & $\begin{array}{r}614.7 * * * \\
(106.7)\end{array}$ & $\begin{array}{r}93.93 * * * \\
(11.90)\end{array}$ & $\begin{array}{r}53.14 * * * \\
(4.586) \\
12.29 * * * \\
(4.384)\end{array}$ \\
\hline & $\begin{array}{r}28 \\
0.664\end{array}$ & $\begin{array}{r}28 \\
0.740\end{array}$ & $\begin{array}{r}28 \\
0.329 \\
\end{array}$ & $\begin{array}{r}28 \\
0.707 \\
\end{array}$ & $\begin{array}{r}28 \\
0.838 \\
\end{array}$ \\
\hline & $\begin{array}{r}(6) \\
\text { Balance }\end{array}$ & $\begin{array}{r}(7) \\
\text { taxes }\end{array}$ & $\begin{array}{r}(8) \\
\text { spending }\end{array}$ & $\begin{array}{r}\text { (9) } \\
\text { UI_spending }\end{array}$ & $\begin{array}{r}(10) \\
\text { UI Spending }\end{array}$ \\
\hline $\begin{array}{l}\text { Change in GDP per } \\
\text { Capita }\end{array}$ & $\begin{array}{r}0.329 * * \\
(0.147)\end{array}$ & $\begin{array}{r}0.470 * * * \\
(0.108)\end{array}$ & $\begin{array}{r}0.140 * * \\
(0.0644)\end{array}$ & $\begin{array}{r}-0.0330 * * * \\
(0.0113)\end{array}$ & \\
\hline $\begin{array}{l}\text { Change in Unemployment } \\
\text { Rate }\end{array}$ & & & & & $\begin{array}{r}34.62 * * * \\
(4.832)\end{array}$ \\
\hline Constant & $\begin{array}{r}-426.7 * * \\
(201.4)\end{array}$ & $\begin{array}{r}-375.7 * * \\
(147.7)\end{array}$ & $\begin{array}{r}50.91 \\
(88.11)\end{array}$ & $\begin{array}{r}42.81^{* *} \\
(15.52)\end{array}$ & $\begin{array}{r}7.040 * * \\
(3.347)\end{array}$ \\
\hline & $\begin{array}{r}25 \\
0.179 \\
\end{array}$ & $\begin{array}{r}25 \\
0.451 \\
\end{array}$ & $\begin{array}{r}25 \\
0.171 \\
\end{array}$ & $\begin{array}{r}25 \\
0.269 \\
\end{array}$ & $\begin{array}{r}25 \\
0.691 \\
\end{array}$ \\
\hline
\end{tabular}




\section{Appendix Table 2:}

Change In Federal Taxes and Spending Per Capita Regressed on Change in State Level Per Capita Income 1996-2007 (i.e. Excluding Great Recession Years)

\begin{tabular}{lrrrr}
\hline \hline & Net Spending & $(1)$ & $(3)$ & $(4)$ \\
& & Taxes & Spending & UI \\
Spending \\
Change in Personal & & & & $-0.00242^{* *}$ \\
Income per capita & $-0.267^{* * *}$ & $0.260^{* * *}$ & -0.00710 & $(0.00120)$ \\
& $(0.0727)$ & $(0.0607)$ & $(0.0457)$ & \\
Constant & 320.4 & 220.3 & $540.6^{* * *}$ & 2.614 \\
& $(246.2)$ & $(165.1)$ & $(174.2)$ & $(3.301)$ \\
& & & & 1176 \\
\end{tabular}

State level annual data 1996-2008. Robust standard errors in parentheses. Net Spending is spending minus taxes. UI is Unemployment Insurance spending. ${ }^{* * *} \mathrm{p}<0.01,{ }^{* *} \mathrm{p}<0.05,{ }^{*} \mathrm{p}<0.1$ 


\section{Appendix Table 3:}

2005 Level of Federal Taxes and Spending Per Capita Regressed on 2005 State Per Capita Income

\begin{tabular}{lrrr}
\hline \hline & $(1)$ & $(2)$ & $(3)$ \\
& Balance & Taxes & Spending \\
Personal Income per & $-0.396^{* * *}$ & $0.395 * * *$ & -0.000970 \\
Capita & $(0.0803)$ & $(0.0509)$ & $(0.0453)$ \\
& & & 32.99 \\
Constant & $13,475^{* * *}$ & $-13,442 * * *$ & $(1,473)$ \\
& $(2,625)$ & $(1,673)$ & 49 \\
& 49 & 49 & 0.000 \\
\hline \hline
\end{tabular}

Robust standard errors in parentheses. ${ }^{* * *} \mathrm{p}<0.01,{ }^{* *} \mathrm{p}<0.05,{ }^{*} \mathrm{p}<0.1$. Excludes Delaware due to large amount of Federal tax paid by corporations legally based in Delaware. 


\section{Appendix Table 4:}

\section{Change in EU Spending and Contributions Per Capita Regressed on change in GDP Per Capita}

\begin{tabular}{lrrr}
\hline \hline & $(1)$ & $(2)$ & $(3)$ \\
& balance & spending & contribution \\
Change in GDP per & & 0.00251 & $0.00885^{* *}$ \\
Capita & -0.00634 & $(0.00374)$ & $(0.00383)$ \\
& $(0.00635)$ & 13.84 & $15.71^{*}$ \\
Constant & -1.862 & $(22.27)$ & $(8.801)$ \\
& $(22.74)$ & 156 & 156 \\
& 156 & 0.032 & 0.146 \\
\hline \hline
\end{tabular}

Data are from Eurostat for 2011. Robust standard errors in parentheses

$* * * \mathrm{p}<0.01,{ }^{* *} \mathrm{p}<0.05,{ }^{*} \mathrm{p}<0.1$ 


\section{Appendix Figure 1}

US Level Time Series Scatterplot of Change in Federal Taxes minus Spending Per Capita Against Change in GDP Per Capita

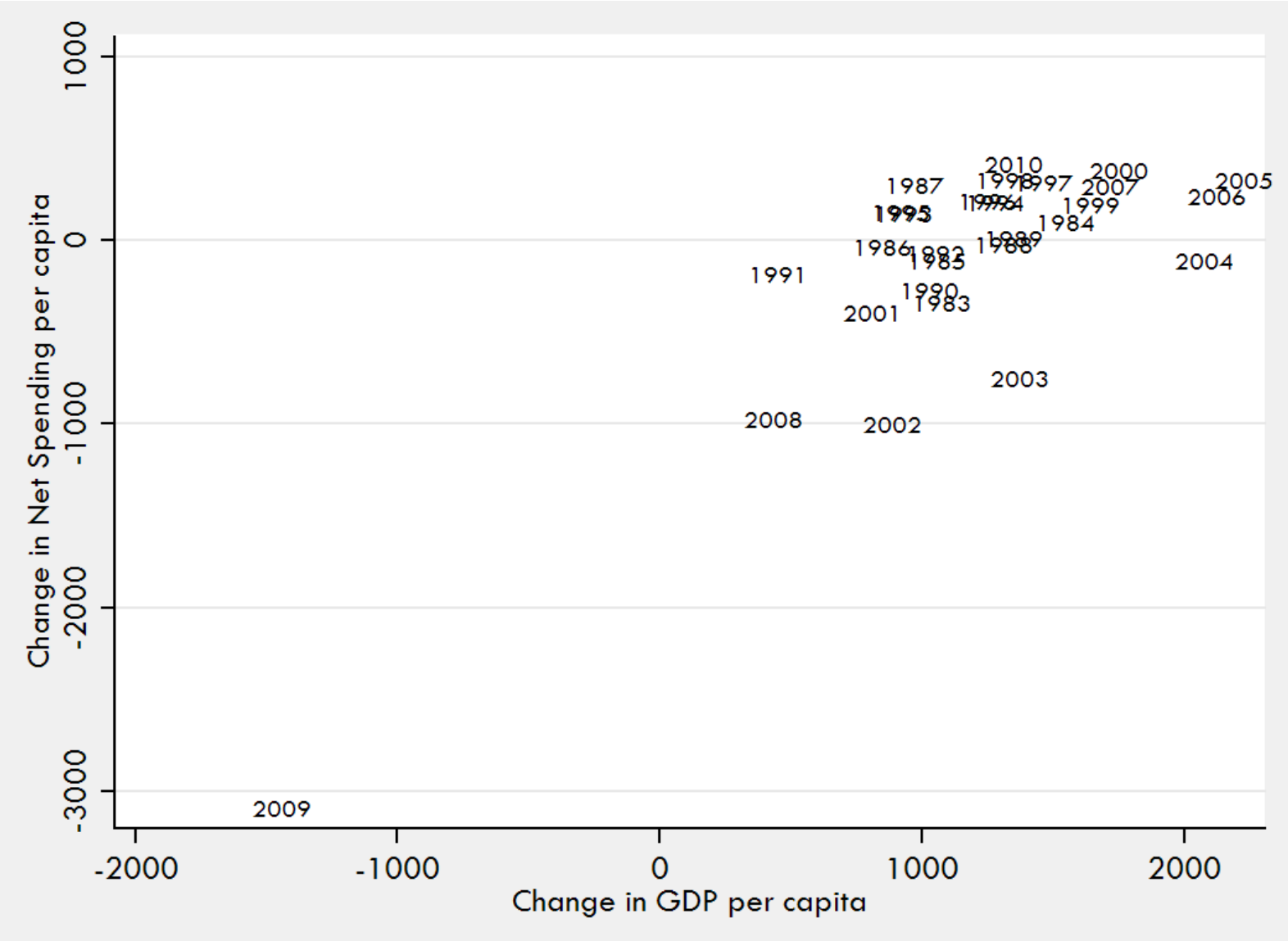


Appendix Figure 2

Histogram of US State Annual Unemployment Changes

Notes: These are annual changes having removed a fixed effect (US time series effect) for each year.

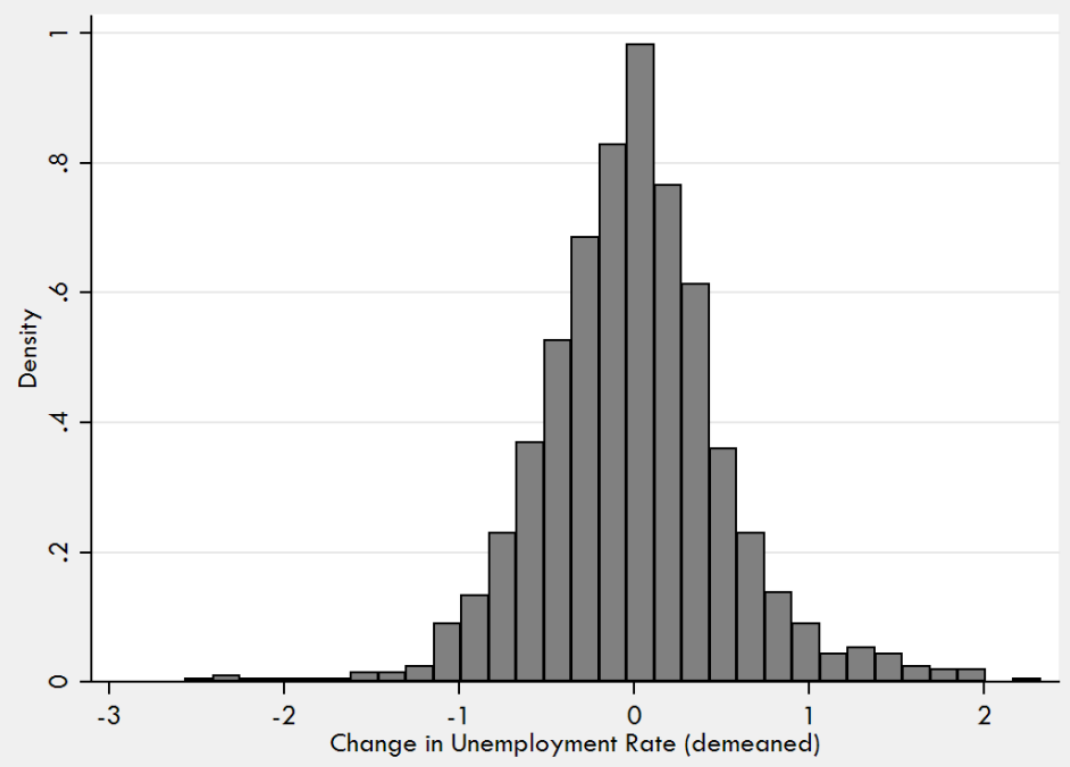


Appendix Figure 3

Histogram of EU Country Annual Unemployment Changes

Notes: These are annual changes having removed a fixed effect for each year.

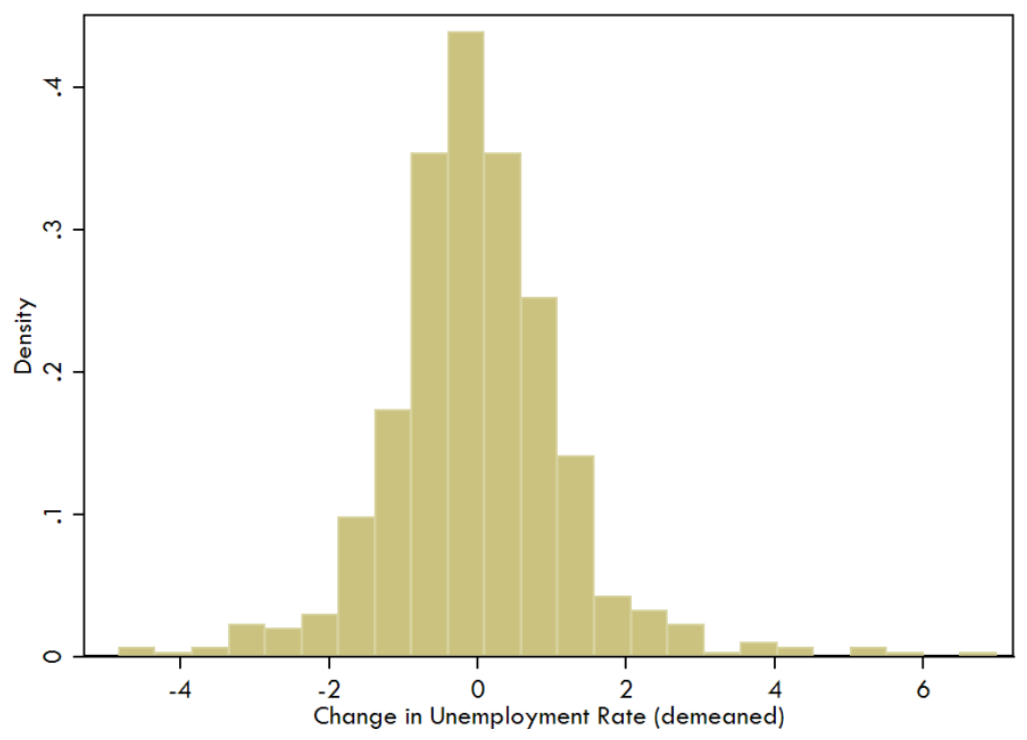

